\title{
9p21 and 13q14 dosages in ependymomas. A clinicopathologic study of 101 cases
}

\author{
Veena Rajaram ${ }^{1}$, Eric C Leuthardt ${ }^{2}$, Pratima K Singh ${ }^{1}$, Jeffrey G Ojemann ${ }^{2}$, Daniel J Brat ${ }^{3}$, \\ Richard A Prayson ${ }^{4}$ and Arie Perry ${ }^{1}$ \\ ${ }^{1}$ Department of Pathology; ${ }^{2}$ Neurosurgery, Washington University School of Medicine, St Louis, MO, USA; \\ ${ }^{3}$ Department of Pathology, Emory University, Atlanta, GA, USA and ${ }^{4}$ Department of Pathology, Cleveland \\ Clinic Foundation, Cleveland, OH, USA
}

\begin{abstract}
Ependymomas are glial neoplasms whose clinical behavior is difficult to predict based on histology alone. Recently, a comparative genomic hybridization study identified frequent chromosome $9 p$ and $13 q$ losses in anaplastic ependymomas, suggesting that p16 and RB alterations may be involved in tumor progression. In order to test this hypothesis further, 101 myxopapillary, conventional, and anaplastic ependymomas (51 spinal and 50 intracranial tumors) were tested for $R B$ and $p 16$ deletions using fluorescence in situ hybridization. Clinical follow-up, ranging from 2 to 198 months (median 46 months), was obtained in 90 cases $(91 \%)$. RB and p16 deletions were seen in 22 of $92(24 \%)$ and 22 of $89(25 \%)$ informative cases, respectively. Polysomies were more frequent in the grade I and II spinal tumors, consistent with prior reports of increased aneuploidy in such cases. No significant genetic associations were seen with tumor grade, recurrence, or death, suggesting that $9 p$ and 13q deletions do not play a prominent role in the malignant progression of ependymomas, as has been implicated in other glioma subtypes.

Modern Pathology (2004) 17, 9-14, advance online publication, 19 November 2003; doi:10.1038/modpathol.3800029
\end{abstract}

Keywords: brain tumor; ependymoma; tumor genetics; FISH; p16/RB; RB pathway; prognosis

Ependymomas are glial neoplasms that constitute $5 \%$ of the central nervous system tumors and are the third most common brain tumors in the pediatric age group. The World Health Organization (WHO) classification of these tumors, based on histology, divides them into grade I (myxopapillary), grade II (conventional), and grade III (anaplastic) ependymomas. Histologic grading of these tumors does not predict clinical behavior as accurately as other gliomas, ${ }^{1-6}$ although there are some studies that show a significant association between tumor grade and prognosis. ${ }^{7-10}$ Thus far, the only consistent prognostic variables include extent of surgical resection, with tumor location and patient's age also having some significance.

Ependymomas have been studied cytogenetically for nearly three decades, although few clues have emerged to elucidate their tumorigenesis and identify potential molecular targets for therapy. ${ }^{11}$ Measures of proliferative activity (Ki-67 labeling index)

Correspondence: A Perry, Division of Neuropathology, Box 8118, Washington University School of Medicine, 660 S Euclid Avenue, St Louis, MO 63110-1093, USA.

E-mail: aperry@pathbox.wustl.edu

Received 13 June 2003; revised 09 October 2003; accepted 14

October 2003; published online 19 November 2003 as well as p53, bcl-2, tenascin, vascular endothelial growth factor (VEGF) and epidermal growth factor (EGFR) protein expression levels have been correlated with tumor grade and clinical behavior, although none of these have become standard ancillary tools in routine diagnostic settings. ${ }^{12,13}$ Chromosomal abnormalities have also been associated with tumor location, grade, and patient's age. ${ }^{14}$ The most common is chromosome 22 deletion, with inactivation of the NF2 gene on $22 q 12$ and loss of its protein product, merlin being specifically implicated in the spinal ependymomas. ${ }^{15-18}$ Data suggest that other 22q-associated genes and other chromosomal regions are likely involved in the intracranial counterparts. Amplification and/or overexpression of mdm2 may also be involved in ependymal tumorigenesis and response of tumor to chemotherapy, ${ }^{19}$ although additional studies are needed to confirm this finding. Other reported abnormalities include gains of chromosome 7 and losses of chromosomes 6, 9, $10,11,13$, and $17 .^{20,21}$

The $p 16^{I N K 4 A}(C D K N 2 A)$ gene on $9 \mathrm{p}$ and the $R B$ gene on $13 q$ are key tumor suppressor genes in a cell cycle regulatory pathway that is commonly inactivated in a wide range of cancer types. The p16 protein inhibits the formation of the cyclin-depen- 
dent kinase 4/cyclin D1 complex, disrupting its ability to phosphorylate and inactivate RB. The RB protein normally prevents the entry of the cell into the S-phase of the cell cycle by blocking E2F transcription factors from activating the genes needed for DNA replication. Thus, a disruption of either $\mathrm{p} 16^{\mathrm{INK} 4 \mathrm{~A}}$ or $\mathrm{RB}$ leads to deregulated cell proliferation and supports tumor progression. ${ }^{22} \mathrm{~A}$ previous study of p16 expression in neuroglial tumors showed that $\mathrm{p} 16^{\mathrm{INK} 4 \mathrm{~A}}$ in ependymomas was expressed only when cellular proliferation had reached a threshold, with recurrent ependymomas having the highest expression levels of $\mathrm{p} 16^{\mathrm{INK} 4 \mathrm{~A}} \cdot{ }^{23} \mathrm{~A}$ recent comparative genomic hybridization (CGH) study identified frequent losses of chromosomes 9 and 13 in anaplastic intracranial ependymomas, suggesting that alterations of this pathway may be critical in the malignant progression of ependymomas, ${ }^{24}$ as they are in the diffuse glioma subtypes, astrocytomas, mixed oligoastrocytomas, and oligodendrogliomas. ${ }^{25-28}$ However, this was a small study and clinicopathologic associations were not thoroughly explored. In the current study, we thus assessed p16 and $R B$ gene dosages by fluorescence in situ hybridization (FISH) in a large cohort of ependymomas and correlated the data with tumor grade, site of disease, patients age, and clinical outcome.

\section{Materials and methods}

\section{Patient/Tumor Cohort}

Archival ependymoma specimens resected between 1990 and 2000 were retrieved from the files of Lauren V Ackerman Laboratory of Surgical Pathology at the Washington University Medical Center in St Louis, MO. All available slides were reviewed and a representative paraffin block was selected per tumor for further study. Sections of $5 \mu \mathrm{m}$ thickness were cut and mounted on poly-L-lysine-coated slides for FISH. Additional cases were obtained as unstained ependymoma tissue microarray (TMA) slides from the Cleveland Clinic and Emory University pathology files. All tumors were classified as WHO grade I (myxopapillary), grade II (conventional), or grade III (anaplastic) ependymomas based on current (WHO) criteria. ${ }^{29}$ Subependymomas were not included in this study. Clinical follow-up was obtained by chart review.

\section{Fluorescence In Situ Hybridization}

Single-and dual-color FISH experiments were performed for $R B$ and paired $p 16$ and CEP9 probes as previously reported. ${ }^{30}$ Following deparaffinization, the sections were subjected to target retrieval by steam cooking in citrate buffer for $20 \mathrm{~min}$ followed by a 20-min cool-down period and a 5-min wash (distilled water). This was followed by pepsin
( $4 \mathrm{mg} / \mathrm{ml}$ ) digestion at $37^{\circ} \mathrm{C}$ for $30 \mathrm{~min}$. The slides were then washed in $2 \times$ SSC and allowed to air dry. Commercially available $R B$ (13q14), p16 (9p21), and CEP9 FISH probes (Vysis, Downers Grove, IL, USA) were utilized. Since no reliable chromosome 13 centromere probes are available (ie, they crosshybridize with centromere 21), single-color $R B$ hybridizations were performed without a reference probe. The probes were diluted in DenHyb hybridization buffer (Insitus Laboratories, Albuquerque, NM, USA) to a concentration of 1:25 $(R B)$ and 1:50 (p16 and CEP9) for whole tissue sections and to a concentration of 1:10 for the TMAs. The hybridization mix (10-20 $\mu \mathrm{l}$ per slide depending on target area) was applied to the sections, followed by simultaneous denaturation of the probe and target DNA at $90^{\circ} \mathrm{C}$ for $13 \mathrm{~min}$. Overnight hybridization at $37^{\circ} \mathrm{C}$ took place in a humidified chamber. Posthybridization washes in $50 \%$ formamide/ $1 \times$ SSC $(5 \mathrm{~min})$ and $2 \times$ SSC $(5 \mathrm{~min})$ were performed at room temperature, and the slides were allowed to air dry. DAPI $(0.5 \mu \mathrm{l} / \mathrm{ml}$; Insitus Laboratories, Albuquers, NM, USA) was used as a nuclear counterstain, and the sections were viewed under an Olympus BX60 fluorescent microscope with appropriate filters (Olympus, Melville, NY, USA).

Sections showing sufficient hybridization efficiency ( $>90 \%$ nuclei with signals) were evaluated, with 100-200 intact non-overlapping nuclei scored for the number of fluorescent signals. The cutoffs for abnormalities/deletions were based on counts from non-neoplastic control specimens (temporal lobectomy specimens for seizure control) for each probe. Interpretation of deletion required $>50 \%$ of nuclei containing one RB or one p16 signal (mean +3 s.d. in controls). Cases with monosomy 9 (one CEP9 and one p16 signal) were similarly considered p16 deleted. Relative p16 deletions were defined by p16:CEP9 ratios $<0.8$ and frequently contained individual nuclei with half as many test signals as reference probe signals (eg, two p16 and four CEP9). Cases with homozygous p16 deletions were recognized by the presence of $>20 \%$ nuclei with CEP9, but no p16 deletions. Adjacent nonneoplastic cells (eg, endothelial cells) served as an internal control to rule out the possibility of partial hybridization failure, given that CEP9 has a higher hybridization efficiency than p16. For the purpose of this study, all FISH deletion types were considered to be biologically equivalent. Since nuclei with $>2$ signals were rarely seen in nonneoplastic controls, polysomies (gains) were arbitrarily defined as $>5 \%$ nuclei containing three or more signals.

Hybridizations were digitally photographed using a high-resolution black and white COHU CCD camera, with a Z-stack motor programmed to capture images at sequential DAPI (one level), FITC (five levels), and rhodamine (five levels) filter settings. Reconstruction into a single superimposed image with blue, green, and red pseudocolors was 
accomplished using a CytoVision ${ }^{\mathrm{TM}}$ basic workstation (Applied Imaging, Santa Clara, CA, USA).

\section{Statistics}

Associations between specific genetic alterations and patient's age (pediatric/adult), grade (I and II/ III), tumor location (intracranial/intraspinal), recurrences (yes/no), and patient's death (yes/no) were evaluated based on the $\chi^{2}$ or Fisher's exact tests, depending on the size of the individual groups being tested. Reported $P$-values $<0.05$ were considered to be statistically significant.

\section{Results}

The study cohort consisted of 101 cases, including 51 spinal ependymomas: nine myxopapillary (WHO grade I) and 42 conventional (grade II). The intracranial tumors (50) included 30 posterior fossa and 20 supratentorial ependymomas, with 32 conventional (grade II) and 18 anaplastic (grade III) examples. The median age was 27 years (range 1-72) for the entire group, 41 years (range 13-69) for the spinal, and 16 years (range 1-72) for the intracranial tumors. There were 36 pediatric patients $(<18$ years) with a median of 7.6 years, presenting with four spinal and 32 intracranial tumors (11 supratentorial, 21 posterior fossa). They were classified as one myxopapillary, 22 conventional, and 13 anaplastic ependymomas. There were 65 adult patients with 47 spinal and 18 intracranial tumors (nine each of supratentorial and posterior fossa tumors). They were classified as eight myxopapillary, 52 conventional, and five anaplastic ependymomas. There were 91 primary and five paired (primary and recurrent) tumors (10 cases). The representative H\&E patterns are shown in Figure 1.

FISH results were interpretable for at least one marker in $99(98 \%)$ cases total, with $92(91 \%) R B$ and $89(88 \%)$ p16 interpretable cases total. The remaining cases were non-informative due to indeterminate signals or tissue loss, the latter primarily a problem in the TMA sections. The results are summarized in Table 1, and representative patterns of FISH are shown in Figure 1.

Deletions of $p 16$ or $R B$ were encountered in $37 \%$ of all tumors and did not vary significantly by site of disease, tumor grade, or patient's age (Tables 1 and 2 ). Of the 27 with $p 16$ deletions, eight $(30 \%)$ had evidence of homozygous deletion. The remainder had loss of only one copy. Polysomies or chromosomal gains were seen more frequently in the myxopapillary and conventional ependymomas than in the anaplastic tumors, particularly those in the spinal cord region. Percentages of polysomic cells ranged from 7 to 76 in individual cases with most in the 20-30 range.

Follow-up was obtained for 90 cases (91\%): 45 spinal ependymomas with a median follow-up period of 3 years, 10 months (range 2-198 months), $27 \mathrm{PF}$ ependymomas with a median follow-up of 2 years, 3 months (range 11 days to 167 months), and 18 ST cases with a median follow-up period of 4 years (range 10-153 months).

In all, 17 patients died, two in the postoperative period; seven (41\%) had deletions for $p 16$ or $R B$ and five $(29 \%)$ had polysomies. A total of 24 patients had recurrences, 8 of them had $p 16$ or $R B$ deletions and four of them had polysomies. In all, 14 patients are alive with residual disease, five of them had $p 16$ or $R B$ deletions and two had polysomies. In all, 41 patients are alive with no evidence of disease, 17 (41\%) with $p 16$ or $R B$ deletions and 24 (59\%) with polysomies. These differences are not statistically significant.

\section{Discussion}

Both losses and gains of chromosomes 9 and 13 have been previously reported in cytogenetic studies of ependymomas. ${ }^{11,21,24,31-34}$ Based on the recent finding of CGH detectable losses in high-grade examples, it was suggested that the RB pathway may be specifically targeted in the process of tumor progression. ${ }^{24}$ In contrast, our larger study similarly identified a subset of ependymomas with $9 p$ or $13 q$ deletions by FISH, but there were no obvious associations with tumor grade, location, patients age, recurrences, or death. Given that relatively few recurrences and deaths occurred during the clinical follow-up period, it may be that a longer follow-up is necessary to exclude entirely associations with biologic behavior. It is also possible that the number of deletions is underestimated by the FISH technique (eg, small deletions or relative $R B$ deletions in an extensively polyploid tumor). However, our frequencies of $9 p$ and $13 q$ losses are similar to those previously reported using other techniques ${ }^{11,14,24,32-34}$ and there appears to be no clear suggestion of a prognostic association based on the available data thus far. Therefore, it is probable that genes other than $p 16$ and $R B$ may be targeted by the $9 p$ and $13 q$ deletions in ependymomas. Also, other mechanisms of gene inactivation, such as promoter hypermethylation, cannot be excluded by cytogenetic methods, such as FISH or CGH. Nonetheless, a study of 16 ependymomas by Bortolotto et $a l^{31}$ did not find any evidence of promoter hypermethylation. ${ }^{31}$ Similar to our study, the involvement of p16 was relatively uncommon, including three immunonegative cases and one case with homozygous deletion. ${ }^{35}$

Polysomies were also observed more frequently in our spinal and low-grade (I and II) neoplasms, consistent with prior observations that the level of aneuploidy/polyploidy is inversely proportional to grade. Contrary to many other tumor types, the anaplastic ependymomas have the fewest and the myxopapillary ependymomas the greatest number of cytogenetic alterations. ${ }^{33,36}$ Polysomies have also 
been reported more frequently in adult than in pediatric tumors, although it is unclear whether this has more to do with age, location, or tumor grade, since the majority of adult ependymomas are low- grade spinal examples. ${ }^{33}$ Polysomies were seen slightly more frequently in our primary tumors without subsequent recurrence than in those with recurrence, although this difference was not statis-
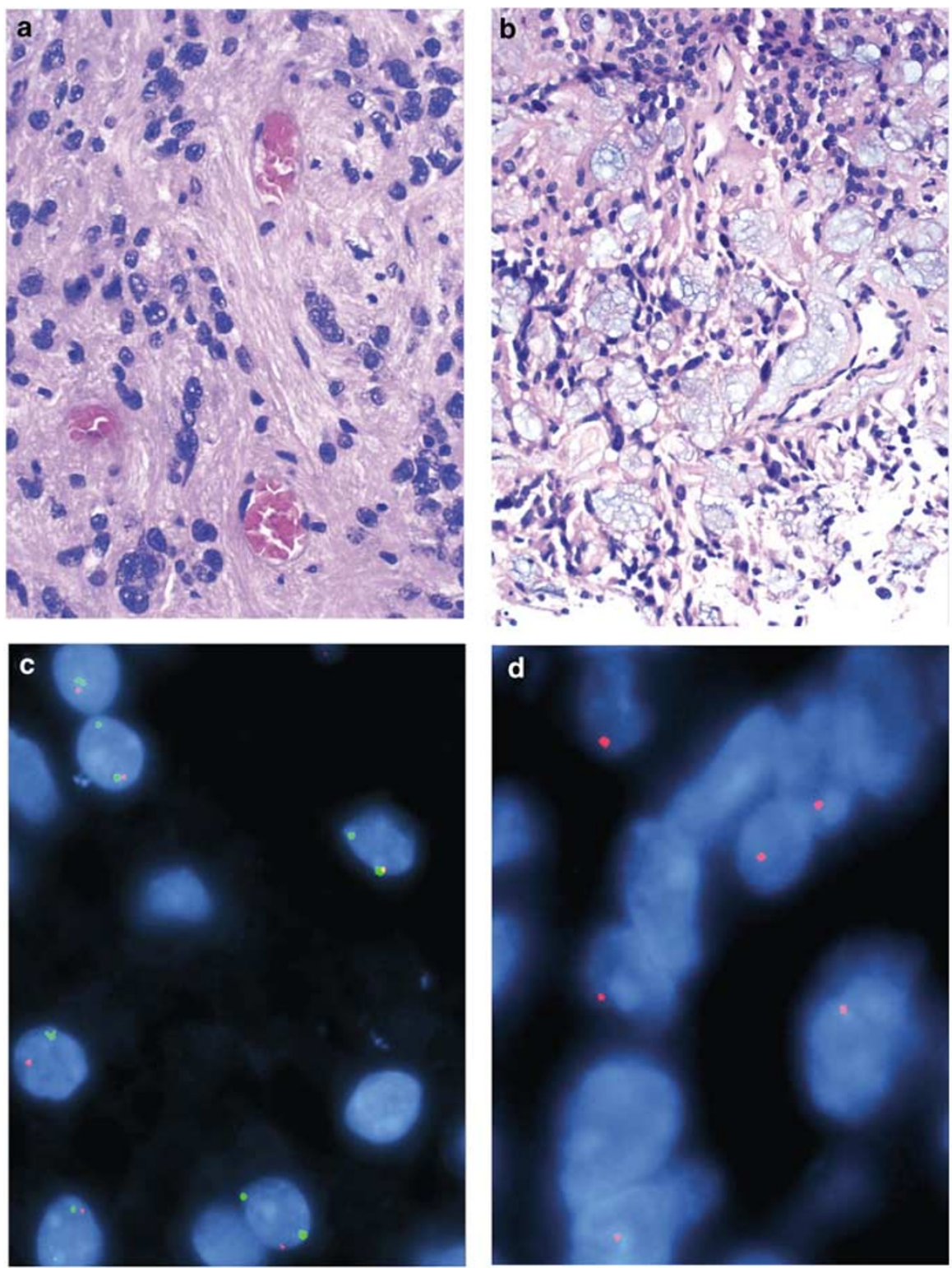

Figure 1 Representative morphologic and FISH images of ependymal tumors: (a) conventional ependymoma (WHO grade II) with perivascular nuclear-free zones or pseudorosettes; (b) Myxopapillary ependymoma (WHO grade I) with prominent mucoid degeneration; (c) FISH image of $p 16$ deletion with two green signals (CEP9) and one red ( $p 16)$ signal in the majority of tumor nuclei; (d) $R B$ deletion with one red $R B$ signal in the majority of nuclei. A few FISH signals are beyond the plane of focus utilized for photography.

Table 1 p16 and $R B$ alterations in ependymomas in relation to location and grade of tumor

\begin{tabular}{|c|c|c|c|c|c|}
\hline & IC & Spinal & $M P E$ & E & $A E$ \\
\hline $\mathrm{RB}$ deletion & $10 / 45(22 \%)$ & $12 / 47(26 \%)$ & $3 / 8(38 \%)$ & $15 / 68(22 \%)$ & $4 / 16(25 \%)$ \\
\hline P16 deletion & $15 / 45(33 \%)$ & $12 / 47(26 \%)$ & $1 / 7(14 \%)$ & $18 / 64(28 \%)$ & $3 / 17(18 \%)$ \\
\hline RB or p16 deletion & $20 / 50(40 \%)$ & $17 / 49(35 \%)$ & $4 / 8(50 \%)$ & $29 / 72(40 \%)$ & $5 / 19(26 \%)$ \\
\hline Polysomies & $11 / 50(22 \%)$ & $21 / 49(42 \%)$ & $4 / 8(50 \%)$ & $23 / 72(32 \%)$ & $5 / 19(26 \%)$ \\
\hline
\end{tabular}

IC, intracranial; MPE, myxopapillary ependymoma; E, conventional ependymoma; AE, anaplastic ependymoma. 
Table $2 p 16$ and $R B$ alterations in pediatric and adult ependymomas

\begin{tabular}{|c|c|c|c|c|}
\hline & RB deletion & p16 deletion & $\mathrm{RB}$ or p16 deletion & Polysomy \\
\hline Pediatric & $7 / 31(23 \%)$ & $10 / 33(30 \%)$ & 13/35 (37\%) & $7 / 35(20 \%)$ \\
\hline Adult & $15 / 61(25 \%)$ & $12 / 56(21 \%)$ & $24 / 64(38 \%)$ & $18 / 64(28 \%)$ \\
\hline
\end{tabular}

tically significant. As in prior studies, we also found that polysomies were equally common in primary and recurrent tumors, but there were few of the latter cases in this study. ${ }^{36,37}$

In conclusion, this study shows that $9 p$ and $13 q$ deletions are seen in a subset of ependymomas, although they do not have obvious associations with tumor grade, age, location, or overall prognosis, as they do in the diffuse gliomas. Therefore, they are not likely to play a prominent role in the malignant progression of ependymomas. Lastly, our data suggest that other chromosome $9 p$ and $13 q$ genes may be involved in the tumorigenesis of this tumor type, besides the $p 16$ and $R B$ genes. Further studies are needed to identify candidate genes within these regions.

\section{References}

1 Fokes Jr EC, Earle KM. Ependymomas: clinical and pathological aspects. J Neurosurg 1969;30:585-594.

2 Mork SJ, Loken AC. Ependymoma: a follow-up study of 101 cases. Cancer 1977;40:907-915.

3 Ross GW, Rubinstein LJ. Lack of histopathological correlation of malignant ependymomas with postoperative survival. J Neurosurg 1989;70:31-36.

4 Schiffer D, Chio A, Giordana MT, et al. Histologic prognostic factors in ependymoma. Childs Nerv Syst 1991;7:177-182.

5 Robertson PL, Zeltzer PM, Boyett JM, et al. Survival and prognostic factors following radiation therapy and chemotherapy for ependymomas in children: a report of the Children's Cancer Group. J Neurosurg 1998;88:695-703.

6 Sala F, Talacchi A, Mazza C, et al. Prognostic factors in childhood intracranial ependymomas: the role of age and tumor location. Pediatr Neurosurg 1998;28: 135-142.

7 Ernestus RI, Schroder R, Stutzer H, et al. Prognostic relevance of localization and grading in intracranial ependymomas of childhood. Childs Nerv Syst 1996;12:522-526.

8 Horn B, Heideman R, Geyer R, et al. A multiinstitutional retrospective study of intracranial ependymoma in children: identification of risk factors. J Pediatr Hematol Oncol 1999;21:203-211.

9 Kovalic JJ, Flaris N, Grigsby PW, et al. Intracranial ependymoma long term outcome, patterns of failure. J Neurooncol 1993;15:125-131.

10 Mansur DB, Michalski JM, Ojemann JG, et al. Longterm results of surgery and post-operative radiation therapy in the curative management of intracranial ependymoma [abstract]. Int J Radiat Oncol Biol Phys 2002;54:204-205.
11 Friede RL, Pollak A. The cytogenetic basis for classifying ependymomas. J Neuropathol Exp Neurol 1978;37:103-118.

12 Bennetto L, Foreman N, Harding B, et al. Ki-67 immunolabelling index is a prognostic indicator in childhood posterior fossa ependymomas. Neuropathol Appl Neurobiol 1998;24:434-440.

13 Korshunov A, Golanov A, Timirgaz V. Immunohistochemical markers for intracranial ependymoma recurrence. An analysis of 88 cases. J Neurol Sci 2000;177:72-82.

14 Hamilton RL, Pollack IF. The molecular biology of ependymomas. Brain Pathol 1997;7:807-822.

15 Ebert C, von Haken M, Meyer-Puttlitz B, et al. Molecular genetic analysis of ependymal tumors. NF2 mutations and chromosome 22q loss occur preferentially in intramedullary spinal ependymomas. Am J Pathol 1999;155:627-632.

16 Lamszus K, Lachenmayer L, Heinemann U, et al. Molecular genetic alterations on chromosomes 11 and 22 in ependymomas. Int J Cancer 2001;91:803-808.

17 Singh PK, Gutmann DH, Fuller CE, et al. Differential involvement of protein 4.1 family members DAL-1 and NF2 in intracranial and intraspinal ependymomas. Mod Pathol 2002;15:526-531.

18 Gutmann DH, Giordano MJ, Fishback AS, et al. Loss of merlin expression in sporadic meningiomas, ependymomas and schwannomas. Neurology 1997;49: 267-270.

19 Suzuki SO, Iwaki T. Amplification and overexpression of mdm2 gene in ependymomas. Mod Pathol 2000;13:548-553.

20 Mazewski C, Soukup S, Ballard E, et al. Karyotype studies in 18 ependymomas with literature review of 107 cases. Cancer Genet Cytogenet 1999;113:1-8.

21 Tong CY, Zheng PP, Pang JC, et al. Identification of novel regions of allelic loss in ependymomas by highresolution allelotyping with 384 microsatellite markers. J Neurosurg 2001;95:9-14.

22 Sherr CJ. The INK4a/ARF network in tumour suppression. Nat Rev Mol Cell Biol 2001;2:731-737.

23 Bouvier-Labit C, Civatte M, Bartoli C, et al. p16INK4a and p19INK4d mRNA expression in neuroglial tumours: correlation with Ki67 proliferation index. Neuropathol Appl Neurobiol 1999;25:408-416.

24 Hirose Y, Aldape K, Bollen A, et al. Chromosomal abnormalities subdivide ependymal tumors into clinically relevant groups. Am J Pathol 2001;158: 1137-1143.

25 Perry A, Anderl KA, Borell TJ, et al. Detection of p16, $\mathrm{RB}, \mathrm{CDK} 4$, and p53 gene deletion/amplification by fluorescence in situ hybridization (FISH) in 96 gliomas. Am J Clin Pathol 1999;112:801-809.

26 Smith JS, Jenkins RB. Genetic alterations in adult diffuse glioma: occurrence, significance, and prognostic implications. Front Biosci 2000;5:213-231.

27 von Deimling A, Fimmers R, Schmidt MC, et al. Comprehensive allelotype and genetic analysis of 466 
human nervous system tumors. J Neuropathol Exp Neurol 2000;59:544-558.

28 Reifenberger G, Louis DN. Oligodendroglioma: toward molecular definitions in diagnostic neuro-oncology. J Neuropathol Exp Neurol 2003;62:111-126.

29 Wiestler OD, Schiffer D, Coons SW, et al. Ependymal tumors. In: Kleihues P, Cavenee WK (eds). Pathology and Genetics of Tumours of the Nervous System. World Health Organization Classification of Tumors. IARC Press: Lyon, 2000, pp. 71-81.

30 Perry A, Fuller CE, Banerjee R, et al. Ancillary FISH analysis for $1 p$ and $19 q$ status: preliminary observations in 287 gliomas and oligodendroglioma mimics. Front Biosci 2003;8:1-9.

31 Bortolotto S, Chiado-Piat L, Cavalla P, et al. CDKN2A/ p16 in ependymomas. J Neurooncol 2001;54:9-13.

32 Dyer S, Prebble E, Davison V, et al. Genomic imbalances in pediatric intracranial ependymomas define clinically relevant groups. Am J Pathol 2002; 161:2133-2141.
33 Jeuken JW, Sprenger SH, Gilhuis J, et al. Correlation between localization, age, and chromosomal imbalances in ependymal tumours as detected by CGH. J Pathol 2002;197:238-244.

34 Kramer DL, Parmiter AH, Rorke LB, et al. Molecular cytogenetic studies of pediatric ependymomas. J Neurooncol 1998;37:25-33.

35 Korshunov A, Golanov A, Timirgaz V. p14ARF protein (FL-132) immunoreactivity in intracranial ependymomas and its prognostic significance: an analysis of 103 cases. Acta Neuropathol (Berl) 2001;102:271-277.

36 Scheil S, Bruderlein S, Eicker M, et al. Low frequency of chromosomal imbalances in anaplastic ependymomas as detected by comparative genomic hybridization. Brain Pathol 2001;11:133-143.

37 Ward S, Harding B, Wilkins P, et al. Gain of 1q and loss of 22 are the most common changes detected by comparative genomic hybridisation in paediatric ependymoma. Genes Chromosomes Cancer 2001; 32:59-66. 